\title{
OSTEOPOROSIS JUVENIL IDIOPÁTICA. PRESENTACIÓN DE UN CASO
}

\author{
Idiopathic juvenile osteoporosis. \\ A case presentation
}

Francisco Carvajal Martínez $z^{a}$ Yadenys Bioti Torres ${ }^{b}$ y Mónica Carvajal Aballe ${ }^{c}$

Recibido: 4 de abril, 2019 • Aprobado: 6 de julio, 2019

Cómo citar: Carvajal Martínez F, Bioti Torres Y, Carvajal Aballe M. Osteoporosis juvenil idiopática. Presentación de un caso. cysa [Internet]. 22 de noviembre de 2019 [citado 25 de noviembre de 2019];3(3):59-3. Disponible en: https://revistas.intec.edu.do/ index.php/cisa/article/view/1538

\section{Resumen}

Introdución: la osteoporosis juvenil idiopática (OJI) es el término descriptivo aplicado a la osteoporosis de etiología desconocida en la edad pediátrica. Se caracteriza por la pérdida progresiva de la masa ósea y el deterioro de su microarquitectura, lo que lleva al aumento de la fragilidad ósea y a la mayor susceptibilidad de fracturas. El principal objetivo del tratamiento de los niños y adolescentes con densidad mineral ósea disminuida es la prevención de las fracturas óseas por fragilidad. Como medidas generales, se recomienda una alimentación equilibrada, con aporte óptimo de calcio y vitamina $\mathrm{D}$ y la promoción de la mayor actividad física posible.

Objetivo: presentar un caso de OJI y enfatizar en aspectos del diagnóstico y tratamiento.

Caso clínico: adolescente, femenina, de 17 años de edad, que acudió al Departamento de Endocrinología Pediátrica del Instituto Nacional de Endocrinología a la edad de 9 ańos por fracturas múltiples desde los 5 años, lo que se

\footnotetext{
a Profesor Titular y Consultante, Investigador Titular y de Mérito, Especialista de segundo grado en Endocrinología. Departamento de Endocrinología Pediátrica, Instituto Nacional de Endocrinología, la Habana, Cuba. Correo-e: endoped@infomed.sld.cu

b Profesor Asistente. Especialista de primer grado en Endocrinología. Especialista de primer grado en Medicina General Integral, Departamento de Endocrinología Pediátrica, la Habana, Cuba
}

\begin{abstract}
Introdution: Juvenile idiopathic osteoporosis (JIO) is the descriptive term applied to osteoporosis of unknown etiology in the pediatric age. It is characterized by the progressive loss of bone mass and the deterioration of its microarchitecture, which leads to increased bone fragility and greater susceptibility to fractures. The main objective of the treatment of children and adolescents with decreased bone mineral density is the prevention of bone fractures due to fragility. As general measures, a balanced diet with an optimal supply of calcium and vitamin $\mathrm{D}$ and the promotion of as much physical activity as possible is recommended.
\end{abstract}

Objective: Present a case of JIO and emphasize aspects of diagnosis and treatment.

Case report: Adolescent, female, 17 years old, who was attended at the Department of Pediatric Endocrinology of the National Institute of Endocrinology at the age of 9 years for multiple fractures since age 5, which was associated with bone pain. Given the symptoms and results of complementary exams, a diagnosis of JIO was confirmed, indicating treatment with calcium and vitamin D. The patient respond favorably, with clinical and

\footnotetext{
${ }^{\mathrm{c}}$ Especialista de primer grado en Oncología Médica, Especialista de primer grado en Medicina General Integral, Máster en Enfermedades Infecciosas, Instituto Nacional de Oncología, la Habana, Cuba
} 
asociaba a dolores óseos. Ante la sintomatología se indicaron los complementarios correspondientes confirmándose el diagnóstico de OJI e indicándose tratamiento con calcio y vitamina $\mathrm{D}$; se evidenció una evolución favorable con mejoría clínica y radiológica, sin necesidad de administrar tratamiento con bifosfonatos.

Palabras clave: osteoporosis; osteoporosis juvenil idiopática; densidad mineral ósea; trastorno del metabolismo fosfocálcico.

\section{Introducción}

La osteoporosis es la enfermedad metabólica ósea más frecuente y se caracteriza por la pérdida progresiva de la masa ósea y el deterioro de su microarquitectura, lo que lleva al aumento de la fragilidad ósea y a la mayor susceptibilidad de en vez de a las fracturas. ${ }^{1,2}$ La misma es erróneamente considerada una enfermedad de las personas mayores debido a su alta prevalencia en la tercera etapa de la vida. Múltiples estudios han evidenciado que, aunque la osteoporosis muestra sus consecuencias en la edad adulta, su origen es pediátrico porque la mayoría de la masa ósea es adquirida durante la etapa de crecimiento. $^{3}$

Es conocido que la masa ósea adquirida al final del crecimiento es un factor determinante del futuro riesgo de fractura; lo que ha despertado en los últimos ańos, gran interés por el estudio de la baja masa ósea/densidad mineral ósea en niños. ${ }^{4}$

Cada vez se encuentra más evidencia de que jóvenes "aparentemente sanos" que presentan fracturas en los antebrazos tienen una baja masa ósea en el sitio de la fractura. De igual forma, estos jóvenes tienen disminución del contenido mineral óseo en otros sitios del esqueleto, como la cadera y la columna vertebral. Esto hace pensar que en cierta población pediátrica existe un mayor riesgo de sufrir fracturas. ${ }^{5}$

En pediatría, y por debajo de los 20 años de edad, se recomienda utilizar el concepto de "densidad radiological improvement, with no need to administer bisphosphonates.

Keywords: Osteoporosis; juvenile idiopathic osteoporosis; bone mineral density; phosphocalcic metabolism disorder.

mineral baja o disminuida para la edad cronológica" cuando los valores de z-score son inferiores a $-2 \mathrm{DE}$, y siguiendo las recomendaciones de la Sociedad Internacional de Densitometría Clínica (ISCD) se definiría la osteoporosis en la edad pediátrica cuando, además de presentar un valor de DMO expresado en puntación z-score y corregido para su edad, sexo y estadio puberal inferior a $-2 \mathrm{DE}$, estén presentes dos de las siguientes condiciones: haber tenido dos o más fracturas de bajo impacto de energía de huesos largos antes de la edad de 10 años; tres o más fracturas en esos mismos huesos antes de la edad de 19 años; $y$, finalmente, una o más fracturas vertebrales a cualquier edad. ${ }^{2,6}$

La osteoporosis juvenil idiopática (OJI) es el término descriptivo aplicado a la osteoporosis de etiología desconocida en la edad pediátrica, es una patología infrecuente con menos de 200 casos descritos desde 1965, con una incidencia estimada de 1:100.000 recién nacidos vivos. ${ }^{7}$ Siendo su primera descripción en el 1938 por Schippers. ${ }^{8}$ La ausencia de manifestaciones clínicas específicas en las fases iniciales de la enfermedad determina, en general, un retraso diagnóstico y su sospecha obliga a un estudio amplio con objetivo de descartar formas secundarias de osteoporosis. ${ }^{9,10}$

Su etiopatogenia es desconocida, aunque se ha observado la existencia de un desequilibrio en la actividad de remodelado óseo, con disminución de la formación ósea de los osteoblastos y actividad normal o discretamente reducida de los osteoclastos, cuyo 
efecto final es el incremento de la reabsorción ósea. La evolución en un alto porcentaje de estos pacientes es hacia la resolución espontánea entre 2 y 4 años, ${ }^{11}$ aunque algunos pacientes desarrollan deformidad progresiva. ${ }^{12}$

La misma se presenta en un nińo hasta el momento sano, con un inicio en la etapa puberal inmediatamente anterior a su comienzo; aunque algunas veces puede iniciarse antes de los 5 años. Es de comienzo insidioso, con síntomas inespecíficos que incluyen dolor óseo, generalmente en la espalda, caderas y pies, dificultad para deambular, disminución de peso y fracturas de huesos largos, vértebras o metáfisis. ${ }^{12,13,14}$

El principal objetivo del tratamiento de los niños y adolescentes con DMO disminuida es la prevención de las fracturas óseas por fragilidad. Como medidas generales, se recomienda una alimentación equilibrada con aporte óptimo de calcio y vitamina $\mathrm{D}$ y la promoción de la mayor actividad física posible. La administración de suplementos farmacológicos debe restringirse a los pacientes con aporte deficitario de estos elementos. En pacientes con movilidad reducida y con grandes discapacidades deben promoverse medidas de fisioterapia y de terapia ocupacional dirigida. ${ }^{15}$

\section{Caso clínico}

Se presenta una adolescente, femenina, de 17 ańos de edad, con el antecedente patológico personal de asma bronquial durante la infancia, siendo tratada con prednisona en varias ocasiones por períodos de hasta 3 meses. A los 5 años comenzó a presentar fracturas frecuentes a diferentes niveles, con un número total de 8 ( 2 en clavícula y codo, metacarpiano, muñeca y aplastamiento de L2-L4). A los 9 años acudió a la consulta de endocrinología pediátrica para estudiar la causa de las fracturas, manifestando, en aquel entonces, dolores óseos, articulares, sobre todo en miembros inferiores; y calambres en ocasiones.

Ante el cuadro de múltiples facturas presentado por la paciente se le realizó estudios de bioquímica, cuyos resultados fueron normales; calcio y fósforo en sangre y orina, encontrando niveles de calcemia disminuida, con hipercalciuria. Se le realizó además TAC de columna lumbar hallando una disminución en la altura del cuerpo vertebral de L1. La radiografía de columna lumbar mostró ligero aplastamiento vertebral de L4. Se le realiza densitometría ósea encontrándose osteoporosis severa en cadera (-4.1 Z-score). El estudio de la paratohormona (PTH) resultó dentro de límites normales. Debido a los antes mencionado se plantea el diagnóstico de Osteoporosis juvenil idiopática y se inicia tratamiento con calcio y vitamina D3. Igualmente, se enfatiza que se cumpla una alimentación rica en calcio.

\section{Evaluación}

En el 2015 se interconsulta con nefrología por la aparición de microlitiasis pequeñas y escasas hacia los grupos caliciales del riñón izquierdo sin repercusión del sistema excretor. Se indica suspender tratamiento sustitutivo y se revalúa en 6 meses por ultrasonido renal sin observase evidencia de litiasis renal, por lo que se reanuda tratamiento con calcio y vitamina D3. No aparición de nuevas fracturas. En abril de 2018 se evalúa respuesta al tratamiento mediante densitometría ósea, encontrándose la misma dentro de parámetros normales. Se mantiene con tratamiento indicado y en seguimiento por consulta externa.

\section{Discusión}

La OJI es el término descriptivo aplicado a la osteoporosis de etiología desconocida en la edad pediátrica. ${ }^{16}$ Solo cuando el estudio exhaustivo descarta la existencia de osteoporosis secundaria se acepta el diagnóstico de OJI. 
En Cuba, el primer caso referido de OJI se recoge por Güell, Carvajal y González en $1973^{17}$, más recientemente, fue publicado otro caso de OJI atípico con alteraciones en la dentición como causa principal, que motivó el estudio del paciente. ${ }^{18}$

En nuestro paciente no se recogen antecedente de terapia medicamentosa con anticonvulsivantes; no existe clínica de enfermedad hematológica u ósea de carácter tumoral, enfermedad gastrointestinal, hepática, malnutrición o nutrición parenteral, entre otras. Se descartan, por el interrogatorio y el cuadro clínico, varias enfermedades que cursan con osteoporosis generalizada, como el mieloma de células plasmáticas, la diabetes mellitus, la inmovilización prolongada, los estados de inmunodeficiencia, la enfermedad de Gaucher, la glucogenosis y otras.

La OJI afecta más a niños que a nińas, siendo de aparición prepuberal y produciéndose únicamente alteraciones óseas. No existe ninguna alteración bioquímica que sea útil en el diagnóstico.

La afectación de los pacientes con OJI es variable, desde formas ligeras de manifestaciones clínico radiológicas hasta otras severas. En las niñas con OJI el pronóstico a largo plazo es incierto, teniendo en cuenta que en estas la masa ósea estará disminuida en relación con la población sana de la misma edad y sexo al culminar la pubertad.

El tratamiento con fármacos antiresortivos como los bifosfonatos ha sido utilizado en nińos con manifestaciones severas de la enfermedad, sobre todo en pacientes con fracturas vertebrales o DMO $<$ a 2,5 DS, en descenso progresivo, y sin respuesta a las medidas convencionales. ${ }^{19}$ Nuestra paciente presentó una evolución satisfactoria con mejoría clínica y radiológica con el tratamiento convencional, por lo que no fue necesaria la administración de bifosfonatos.

62 | Ciencia y Salud 2019; 3(3): 59-63 • Casos clínicos

\section{Bibliografía}

1. Brunetto O.H. Osteoporosis in Childhood. Rev Argent Endocrinol Metab. 2006;43: 90-108.

2. Vrinda S, Wolfgang H. Osteoporosis in children: diagnosis and management. European Journal of Endocrinology.2015;173: R185R197. DOI: 10.1530/EJE-14-0865.

3. Correa M, Rueda B, González E, Navarro CF, Schmidt-RioValle J. Los niveles de mineralización ósea están influenciados por la composición corporal en niños y adolescentes. Nutr Hosp. 2014;30: 763-8.

4. Bianchi ML. Osteoporosis in children and adolescents. Bone. 2007; 41: 486-95.

5. Céspedes C, Wandurraga EA. Enfoque y manejo de la osteoporosis en niños y adolescentes. Univ Méd Bogotá (Colombia). 2008;49(3): 373-87.

6. Bishop N, Arundel P, Clark E, Dimitri P, Farr J, Jones $\mathrm{G}$ et al. International Society of Clinical Densitometry. Fracture prediction and the definition of osteoporosis in children and adolescents. J Clin Densitom. 2014;17: 275-80.

7. Cimaz R, Guez S. Diagnostic et traitement de l'ostéoporose juvénile. Arch Pediatr. 2005;12: 585-93. Available from: http://dx.doi.org/10. 1016/j.arcped.2005.02.007

8. Sanchis V, Martin JA, Salido M, Laguia M. Osteoporosis Idiopática Juvenil (A propósito de un caso). Rev Esp de Cir Ost. 1990: 57-62.

9. Yeste DM, Clemente A, Carrascosa. Osteoporosis juvenil idiopática. Anales de pediatría. 2013;79(3): 200-202.

10. Boyce AM, Gafni RI. Approach to the child with fractures. [Internet]. J Clin Endocrinol Metab. 2011;96: 1943-1952. Available from: http://dx.doi.org/10.1210/jc.2010-2546 
11. Yeste D, Clemente M, Campos A, Fábregas A, Soler L, Carrascosa A. Osteoporosis en pediatría. Rev esp endocrinol pediatr 2017;8 suppl(1): 73-85. Available from: doi. 10.3266/revespendocrinolpediatr.pre2017.apr.389

12. Saggese G, Baroncelli GI, Bertelloni S. Osteoporosis in children and adolescents: diagnosis, risk factors, and prevention. J Pediatr Endocrinol Metab. 2001;14: 833-59.

13. Van der Sluis IM, de Muinck S.Osteoporosis in chilhood: bone density of children in health an disease. J Pediatr Endocrinol Metab. 2001;14: 817-32.

14. Hernández I, Bustamante $M$, Rodríguez $M$, León S, García A. Caso atípico de osteoporosis idiopática juvenil. Rev Cub Ped. 2014;86(1): 108-14.

15. Ward K, Alsop C, Caulton J, Rubin C, Adams J, Mughal Z. Low magnitude mechanical loading is osteogenic in children with disabling conditions. J Bone Miner Res. 2004;19: 360-9.
16. Lorenc R.S. Idiopathic juvenile osteoporosis. Calcif Tissue Int. 2002;70: 395-397. Available from: http://dx.doi.org/10.1007/s00223-0010045-yMedline.

17. Güell R, Carvajal F, González J. Osteoporosis Juvenil Idiopática. Presentación de un caso. Rev. Cub. Ped. 1973;45(mayo-junio): 327-32.

18. Hernández I, Bustamante $M$, Rodríguez $M$, León S, García A. Caso atípico de osteoporosis idiopática juvenil. Rev Cub Ped. 2014;86(1): 108-14.

19. Steelman J, Zeitler P. Pediatric osteoporosis. International Symposium on a Current Review of Pediatric Endocrinoloy. Montreal, Quebec, Canadá 2001; 99-107. 